\title{
Can we design drugs for HIVIAIDS that are less susceptible to resistance?
}

\author{
"The major roadblock for both therapy and prevention of HIV/AIDS is the genetic \\ diversity of the virus."
} Keywords: antiretroviral therapy $\bullet$ molecular mechanisms of drug resistance $\bullet$ rational drug
design $\bullet$ resistance testing and interpretation

Antiretroviral drugs are likely to offer the major treatment and prevention option for HIV/AIDS for the foreseeable future. HIV/AIDS is a serious pandemic. About 35 million people are infected by HIV worldwide and nearly 40 million have died of AIDS related diseases since 1981, according the WHO statistics [1]. An effective vaccine has proved elusive because the virus infects the cells of the immune system. Recent clinical trials, however, have been promising for a broadly neutralizing antibody [2]. In the absence of a vaccine, a number of antiviral drugs have been developed and used in AIDS therapy since 1987. These drugs target essential steps in the viral life cycle, including virus entry and fusion with the host cell, and the viral enzymes, protease, reverse transcriptase and integrase [3]. The majority of drugs inhibit the HIV reverse transcriptase and protease. Different combinations of the 25 US FDA-approved drugs are employed in the standard clinical regimens known as highly active antiretroviral therapy (HAART) or combination antiretroviral therapy (cART). These treatment regimens employ three or more antiviral agents targeting at least two of the viral proteins. This therapeutic approach has proved successful in reducing the viral load and extending the life of infected individuals. Additionally, in recent years, antiretroviral drugs have been given as a preventive measure (pre-exposure prophylaxis or PrEP) to individuals at high risk of infection [4].

The current initial treatment options recommended for drug-naive patients consist of an integrase inhibitor or the protease inhibi- tor, darunavir, combined with two reverse transcriptase inhibitors. These combinations are well tolerated and limit the development of resistance. The recommended preventive treatment of PrEP consists of one or two reverse transcriptase inhibitors, tenofovir and emtricitabine. Ongoing clinical studies are assessing the effectiveness of PrEP, although infection with drug-resistant virus is a potential problem [4].

Antiviral therapy does not provide a cure for AIDS. The virus persists in a latent form because HIV integrates into the host genome, leading to viral reservoirs that are tricky to eradicate. Viral reservoirs in the central nervous system and other inaccessible tissues are particularly problematic for eradication. Even with effective therapy, HIV-infected individuals suffer from a dysfunctional immune system and are susceptible to cardiovascular, kidney, liver and neurological disorders.

The major roadblock for both therapy and prevention of HIV/AIDS is the genetic diversity of the virus. The genetic diversity and rapid selection of variants enable the virus to escape the immune system and hinder the development of effective vaccines as well as limiting drug therapy by resistant mutations [5]. The genetic diversity of HIV arises from the low fidelity of viral replication due to the error prone reverse transcriptase coupled with a high rate of replication and rapid turnover of infected cells [6,7]. Each infected individual may contain a complex mix of evolving viral strains with a total estimated population of about $10^{10} \mathrm{HIV}$ virions per day. In addition to the most com-

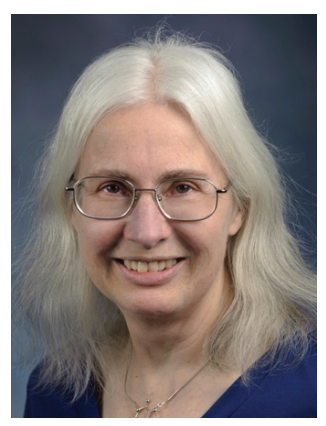

Irene T Weber

Department of Biology, Georgia State University, PO Box 4010, Atlanta, GA 30302-4010, USA

Tel.: +1 4044135411

Fax: +1 4044135301

iweber@gsu.edu 
mon HIV type 1 and the rarer type 2, there are four groups within HIV-1 (M, O, N and P) with distinct geographical distributions. The genetic diversity combined with the high rate of mutations leads to the rapid development of viral resistance. A list of current mutations associated with resistance to the antiviral drugs is available and updated regularly [8]. Drug-resistant species will survive under drug selection pressure and pose a serious challenge for long-term therapy. The same problem of genetic diversity is a major impediment to the development of an effective vaccine.

\section{"Current treatment guidelines recommend resistance testing for newly infected patients or those failing drug therapy."}

Several avenues are available to tackle the challenge of drug resistance. Two important aspects will be considered here: how resistance testing with computational analysis can direct the choice of effective treatment, and how knowledge of the molecular mechanisms of resistance can improve the design of new drugs.

\section{Resistance testing \& interpretation}

Current treatment guidelines recommend resistance testing for newly infected patients or those failing drug therapy. Resistance can be tested in a genotype assay or a phenotype assay. In the phenotype assay, the viral genome is sequenced to identify mutations associated with resistance. In the phenotype assay, viral replication using clinically derived RNA is measured in the presence of different drugs. The genotype assay is preferred due to its lower cost and faster availability of the results. Drug resistance can be predicted from genotype data by two general techniques: rule-based genotype interpretation systems and machine learning algorithms [9]. The majority of prediction methods are genotype interpretation systems. These methods calculate a score for resistance based on the presence of mutations associated with drug resistance in the sequence data. Publicly available examples include HIVdb, ANRS and HIV-GRADE. One important limitation is that this technique depends on the knowledge of predefined drug resistance mutations for the interpretation and unique mutations cannot be evaluated. The alternative approach applies machine learning methods to infer knowledge from different information, such as genotype data, viral load, CD 4 count and phenotype assays for resistance. Two examples are geno2pheno and EuResist. A variety of machine learning methods have been employed, such as Support Vector Machine, logistic regression, Bayesian networks and alternating decision trees. The general limitation of machine learning predictions is the requirement for large amounts of data. The great advantage of these prediction methods is that they do not rely on rules based on known mutations and can be applied to sequences containing novel mutations. Incorporating additional, independent information in the predictions is likely to improve the accuracy. Improved accuracy can be achieved by incorporating the 3D structures of target proteins in a unified sequence-structure representation for machine learning [10]. Additional clinical data can be included. High-throughput 'nextgeneration sequencing' technologies provide a snapshot of the entire viral population of an infected individual at a specific time point [11]. These sequencing techniques combined with improvements in the interpretations of genotype data are likely to lead to a revolution in monitoring drug resistance, selecting the optimal treatments, and ultimately understanding how to overcome viral resistance with novel drugs.

\section{Molecular mechanisms of drug resistance}

Studies of the molecular mechanisms of drug resistance provide invaluable information for the design of improved inhibitors. The current HIV drug targets and molecular mechanisms for resistance have been summarized in [3]. Different drugs elicit distinct patterns of resistance mutations [8]. Mutations often lower the viral fitness, which is the capacity of the virus to produce infectious virions. Initial mutations conferring resistance to drugs often show defects in viral replication. As drug resistance evolves, however, subsequent compensatory mutations will tend to increase viral fitness. Consequently, high levels of resistance may require multiple mutations in the viral genome. Mutations associated with drug resistance can alter the $3 \mathrm{D}$ structure, stability and dynamics of the target protein, causing loss of susceptibility to the inhibitor. The majority of the research on the molecular effects of the mutations has focused on the enzyme inhibitors of the reverse transcriptase, protease and integrase.

The reverse transcriptase inhibitors were the first to be employed in AIDS therapy. The reverse transcriptase is catalytically active as a heterodimer with both subunits encoded by the same gene. The longer p66 subunit comprises the polymerase and C-terminal RNase H domains, while the shorter p51 subunit consists of the polymerase [6]. The two subunits exhibit different conformations of their subdomains, p51 is relatively rigid and p66 is more flexible, forming different conformational arrangements during the course of the polymerase reaction. The clinical inhibitors of the reverse transcriptase are classified as nucleoside reverse transcriptase inhibitors (NRTIs) and non-NRTIs (NNRTIs). NRTIs bind at the polymerase active site 
and are incorporated into the viral DNA, where they block further elongation of the DNA. NNRTIs bind to an allosteric site on the enzyme and inhibit indirectly by stabilizing a catalytically noncompetent conformation of the reverse transcriptase. The mutations and mechanisms associated with resistance are described in $[3,12,13]$. Resistance to NNRTIs can evolve rapidly because the residues forming the binding site are not essential for the polymerase activity. Mutations associated with resistance to NRTIs may alter the binding site for the triphosphate NRTI or enhance the excision of the 'mis'-incorporated NRTI. Resistance mutations may also alter the conformational dynamics of the enzyme. One approach to improved inhibitors is to design compounds with similar interactions with the polymerase as seen for the natural nucleotides. Overall, despite the existence of resistant variants, the NRTIs remain the mainstays of antiviral therapy and prevention via PrEP.

The inhibitors of the HIV protease were first introduced in therapy in 1995, resulting in better suppression of virus replication and increased survival of HIVinfected individuals. The major metabolic side effects of protease inhibitors arising from unwanted inhibition of cellular proteins include lipodystrophy syndrome and insulin resistance. The protease is responsible for proteolytic processing of the viral Gag and Gag-Pol precursor polyproteins in the maturation stage after the virus particle has budded from the host cell [14]. The processes of viral maturation and assembly are tightly coupled [15]. Mutations in the protease and in its polyprotein substrates can interfere with viral replication, producing noninfective viral particles. The mature protease is active as a dimer of chemically identical subunits. The protease is tractable for a variety of studies due to its small size of 99 amino acids per subunit, and the ease of obtaining structural data. Individual mutations associated with drug resistance can alter the inhibitor binding site, the dimer stability or dynamics of the flexible flaps covering the bound inhibitor [16]. Multiple mutations accumulate in the protease gene as resistance evolves. High-level resistance to inhibitors is observed in protease variants with up to 20 mutations. Generally, these mutants lose interactions with inhibitor or substrate, and can show an expanded binding site with increased mobility of the flaps. One promising strategy to overcome resistance is the design of novel inhibitors incorporating groups that provide additional interactions with the backbone atoms of the protease [17]. The potent antiviral inhibitor, darunavir, was designed to introduce new hydrogen bonds with the backbone amides and carbonyl oxygens of the protease. Darunavir is recommended for initial therapy; it has picomolar affinity for the wild-type protease, low toxicity, and retains high affinity for the majority of mutants. This design approach can be extended to target conserved regions of the protease, including the flexible flaps. A recent inhibitor designed by this strategy incorporates fluorines to improve lipid solubility and shows improved penetration of the blood-brain barrier [16]. Effective penetration of the central nervous system with a drug developed from this compound would help to eradicate the viral reservoirs.

\section{"...current efforts focus on development of antiviral inhibitors with favorable bioavailability, pharmacokinetics and ideally, low cost..."}

The HIV integrase performs the essential step of inserting the viral genome into the host cell DNA. Inhibitors targeting the HIV integrase are a more recent development [18]. Integrase inhibitors, or integrase strand transfer inhibitors (INSTIs), were first introduced in 2007. The INSTIs have low toxicity and are now recommended for initial AIDS therapy. Due to their recent introduction, less is known about the mutations associated with resistance [3,19]. However, dolutegravir is highly potent with favorable pharmacokinetic properties and has selected for few resistance mutations compared with the other two available INSTIs. This compound is promising for targeting HIV reservoirs as well as controlling the development of resistance.

\section{Strategies to overcome the challenge of HIV resistance}

General strategies proposed to overcome the problem of resistance include: improved analysis of the viral genome and HIV population in infected individuals [9]; development of a broadly neutralizing antibody [2]; and design of better inhibitors for the current targets or for novel drug targets in viral replication. The design goals for inhibitors targeting resistant variants of the viral enzymes are: high affinity binding to the active site; new interactions with backbone and conserved residues; and stabilization of the catalytically competent conformation of the enzyme. In addition, current efforts focus on development of antiviral inhibitors with favorable bioavailability, pharmacokinetics and ideally, low cost. Better knowledge of the molecular mechanisms for resistance, derived from structural studies and related biophysical and biochemical analyses, will open new possibilities for inhibitors designed to target resistant HIV [3]. One effective strategy is to design compounds that incorporate new interactions with conserved structural regions of the enzymes in order 
to limit the effect of mutations. Novel targets in the viral life cycle include the viral precursor polyproteins and viral interactions with host proteins. Maturation inhibitors have been developed to bind the protease cleavage site in the Gag precursor, although progress has been disappointing to date [16]. The interaction of the HIV integrase with LEDGF/p75 has been targeted by novel inhibitors [20].

In future, a combination of existing and new drugs will be employed for both prevention and treatment of HIV infections. Drug resistance can be suppressed by resistance testing with computational guidance

\section{References}

1 WHO

www.who.int/mediacentre/factsheets/fs360/en/

2 Caskey M, Klein F, Lorenzi JC et al. Viraemia suppressed in HIV-1-infected humans by broadly neutralizing antibody 3BNC117. Nature 522, 487-491 (2015).

3 Menéndez-Arias L. Molecular basis of human immunodeficiency virus type 1 drug resistance: overview and recent developments. Antiviral Res. 98 (1), 93-120 (2013).

4 Wilton J, Senn H, Sharma M et al. Pre-exposure prophylaxis for sexually-acquired HIV risk management: a review. HIV AIDS (Auckl.) 7, 125-36 (2015).

5 Lloyd SB, Kent SJ, Winnall WR. The high cost of fidelity. AIDS Res. Hum. Retroviruses 30 (1), 8-16 (2014).

6 Hu WS, Hughes SH. HIV-1 reverse transcription. Cold Spring Harb. Perspect. Med. 2(10), a006882 (2012).

7 Santoro MM, Perno CF. HIV-1 genetic variability and clinical implications. ISRN Microbiol. 2013, 481314 (2013).

8 Wensing M, Calvez V, Gunthard HF et al. 2014 update of the drug resistance mutations in HIV-1. Top. Antivir. Med. 22, 642-650 (2014).

9 Prosperi MC, De Luca A. Computational models for prediction of response to antiretroviral therapies. AIDS Rev. 14(2), 145-53 (2012).

10 Yu X, Weber IT, Harrison RW. Prediction of HIV drug resistance from genotype with encoded three-dimensional protein structure. BMC Genom. 15(Suppl. 5), S1 (2014). for treatment and the development of high affinity inhibitors retaining effectiveness on resistant variants.

\section{Financial \& competing interests disclosure}

The author's research is supported in part by the grant U01 GM062920 awarded by the NIH. The author has no other relevant affiliations or financial involvement with any organization or entity with a financial interest in or financial conflict with the subject matter or materials discussed in the manuscript apart from those disclosed.

No writing assistance was utilized in the production of this manuscript.

11 Van Laethem K, Theys K, Vandamme AM. HIV-1 genotypic drug resistance testing: digging deep, reaching wide? Curr. Opin. Virol. 14, 16-23 (2015).

12 Das K, Arnold E. HIV-1 reverse transcriptase and antiviral drug resistance. Part 1. Curr. Opin. Virol. 3(2), 111-118 (2013).

13 Das K, Arnold E. HIV-1 reverse transcriptase and antiviral drug resistance. Part 2. Curr. Opin. Virol. 3(2), 119-128 (2013).

14 Konvalinka J, Krausslich HG, Muller B. Retroviral proteases and their roles in virion maturation. Virology 479-480C, 403-417 (2015).

15 Lee SK, Potempa M, Swanstrom R. The choreography of HIV-1 proteolytic processing and virion assembly. J. Biol. Chem. 287(49), 40867-40874 (2012).

16 Weber IT, Kneller DW, Wong-Sam A. Highly resistant HIV-1 proteases and strategies for their inhibition. Future Med. Chem. 7, 1023-1038 (2015).

17 Ghosh AK, Anderson DD, Weber IT et al. Enhancing protein backbone binding - a fruitful concept for combating drugresistant HIV. Angew. Chem. Int. Ed. Engl. 51(8), 1778-802 (2012).

18 Malet I, Calvez V, Marcelin AG. The future of integrase inhibitors of HIV-1. Curr. Opin. Virol. 2(5), 580-587 (2012).

19 Mesplède T, Wainberg MA. Resistance against integrase strand transfer inhibitors and relevance to HIV persistence. Viruses 7(7), 3703-3718 (2015).

20 Christ F, Debyser Z. The LEDGF/p75 integrase interaction, a novel target for anti-HIV therapy. Virology 435(1), 102-109 (2013). 